\title{
UK consensus on pregnancy in multiple sclerosis: 'Association of British Neurologists' guidelines
}

\author{
Ruth Dobson, ${ }^{1,2}$ Pooja Dassan, ${ }^{3,4}$ Megan Roberts, ${ }^{5}$ Gavin Giovannoni, ${ }^{2,6}$ \\ Catherine Nelson-Piercy, ${ }^{7,8}$ Peter A Brex ${ }^{9}$
}

\begin{abstract}
${ }^{1}$ Preventive Neurology Unit, Wolfson Institute of Preventive Medicine, Queen Mary University of London, London, UK

${ }^{2}$ Department of Neurology, Barts Health NHS Trust, Royal London Hospital, London, UK ${ }^{3}$ Department of Neurology, Imperial College Healthcare NHS Trust, London, UK

${ }^{4}$ Department of Neurology, Ealing Hospital, London North West Healthcare NHS Trust, London, UK

${ }^{5}$ MS Trust, London, UK

${ }^{6}$ Blizard Institute, Queen Mary University of London, London, UK

${ }^{7}$ Department of Obstetric Medicine, Guy's and St Thomas' NHS Foundation Trust, London, UK

${ }^{8}$ Queen Charlotte's Hospital, Imperial College Healthcare Trust, London, UK

${ }^{9}$ Department of Neurology, King's College Hospital NHS Foundation Trust, London, UK
\end{abstract}

\section{Correspondence to}

Dr Ruth Dobson, Preventive Neurology Unit, Wolfson Institute of Preventive Medicine, Queen Mary University of London, London E1 2AT, UK; ruth.dobson@qmul.ac.uk

Accepted 30 September 2018

Published Online First

5 January 2019

\section{Check for updates}

(c) Author(s) (or their employer(s)) 2019. No commercial re-use. See rights and permissions. Published by BMJ.

To cite: Dobson R, Dassan $\mathrm{P}$, Roberts M, et al. Pract Neurol 2019;19:106-114.

\begin{abstract}
Multiple sclerosis (MS) is more common in women than men and is most commonly diagnosed in early adulthood; thus, many patients will not have completed their families at the time of diagnosis. There is increasing awareness of the importance of early treatment in preventing long-term disability in MS. Delaying treatment until women with MS have completed their families can lead to the development of irreversible disability in at least some cases. It is therefore important to discuss family planning and pregnancy proactively. However, to date there is limited evidence to inform such discussions. We set out to develop consensus guidelines for the treatment of MS in pregnancy to encourage and facilitate discussions in this important area. The guidelines draw on available evidence from drug-specific pregnancy registers and published literature and have been scored by a panel of experts from a variety of disciplines using modified Delphi criteria. They cover prepregnancy counselling, management during pregnancy, delivery and anaesthetic options, postpartum advice and specific advice regarding currently licensed disease-modifying drugs. As the complexity and range of available disease-modifying drugs increase, further data gathering via a UK-wide MS pregnancy register is recommended.
\end{abstract}

\section{INTRODUCTION}

Multiple sclerosis (MS) is most commonly diagnosed in young women, many of whom will still wish to have children. It is therefore important that family planning and pregnancy is proactively discussed, particularly when considering diseasemodifying treatments. As many as one in six pregnancies in the UK is unintended (UK Office for National Statistics); this must also be taken into account when treating women of childbearing age.
The relatively modest efficacy of many of the early disease-modifying drugs (interferon beta (IFN-B) preparations and glatiramer acetate (GA)), along with limited evidence of safety in pregnancy, has previously led many women to defer starting disease-modifying drugs until after they had completed their families. ${ }^{1}$ In some cases, particularly those for whom conception takes longer than anticipated, this has the potential to lead to a significant delay in starting treatment. In recent years, there has been an increasing appreciation that neuroaxonal damage starts early in relapsing-remitting MS. This damage accrues over time and is the likely cause of the progressive disability that occurs later in the condition. Early intervention with disease-modifying drugs has been shown to reduce/delay long-term disability. ${ }^{2}$ In addition, with the increase in availability of highly active diseasemodifying drugs to control MS, the benefits associated with early treatment appear to be increasing. ${ }^{2}$

However, early treatment causes a dilemma as to what to do when people with MS want to become pregnant while taking disease-modifying drugs or become pregnant after starting this treatment. The overwhelming majority of medications used in MS are FDA category $\mathrm{C}$ for use in pregnancy ('Risk not ruled out'; no studies in humans but potential benefits may warrant use of the drug in pregnant women), as there are limited data on both safety profiles during pregnancy and risks to the developing fetus. An exception is Copaxone (GA), which is FDA category B ('No risk in other studies'; either no risk in animal studies or no risk demonstrated in controlled studies in pregnant women). The summary of product characteristic documentation for GA, IFN-B preparations, dimethyl fumarate and natalizumab 
each state that the treatment should be used in pregnancy only if the benefits outweigh the risks.

While there have been few clinical trials of MS disease-modifying drugs conducted in pregnant women, there is now a significant body of clinical experience of women who have been exposed to treatment during pregnancy. This, in addition to the known pharmacology of these drugs, enables us to draw some conclusions about safety. Pharmacological companies maintain internal databases with data on drug exposures during pregnancy; however these are focused on a single drug of interest, do not prospectively recruit all patients who become pregnant on therapy, and most recorded pregnancies in these databases have only first trimester exposure to the drug.

The risks associated with stopping treatment also need to be considered. The natural reduction of relapses that occurs during pregnancy may not be sufficient to control disease activity, particularly in those with highly active disease. Withdrawal of some of the more highly efficacious disease-modifying drugs has been associated with a return of disease activity and rebound. ${ }^{3}$

For some of the symptomatic treatments, such as antidepressants, guidelines for the general population (which are equally applicable to people with MS) are available to help balance the risk vs benefit of treating during pregnancy. We plan to develop further guidelines bringing together guidance on the safety of using the main symptomatic treatments in pregnancy.

The aim of these consensus guidelines is to encourage and facilitate discussions about family planning and pregnancy between women with MS and their clinicians. They are not meant as a comprehensive review of the literature but where evidence is available, references are provided.

\section{METHODS}

In May 2016, a multidisciplinary working group, all with an interest in the management of MS during pregnancy, met to review the current evidence. Following this, a steering group (PB, PD and MR) met in July 2016 to summarise the discussion into a series of statements. These were then circulated to the working group and two patient representatives using a modified Delphi scoring system. For each statement, the respondent scored that they strongly agreed, agreed, were neutral, disagreed or strongly disagreed. During the first round, they were encouraged to modify the wording of each statement. The steering group then reviewed and revised the statements and they were sent out again for a second round of scoring in January 2017. Consensus was judged to have been agreed if $80 \%$ of the group scored that they strongly agreed or agreed with the statement. The guidelines were reviewed and updated by the authors of this paper in early 2018 before circulation to the working group. The Association of British Neurologists MS Advisory
Group then reviewed the guidelines and their feedback was incorporated into the final manuscript.

\section{Consensus guidelines (figure 1)}

Prepregnancy counselling

Recommendations

- At or soon after diagnosis, all women with MS of childbearing age should have prepregnancy counselling and this should be repeated at regular intervals (at least annually) particularly for those who are on or considering starting medication.

- Women with MS should be advised that if they are considering pregnancy they should discuss this with their MS healthcare professional before trying to conceive.

- Healthcare professionals should consider the safety of all drug treatments during pregnancy when prescribing to women of childbearing age.

Men and women with MS should be advised that

- Having MS does not affect fertility (or risk of miscarriage) and contraception should be used if pregnancy is undesirable. $^{4}$

- MS and/or drugs used in its treatment can cause sexual dysfunction, either directly (eg, loss of libido, erectile dysfunction, vaginal dryness, inability to achieve orgasm) or indirectly through other symptoms of MS (eg, bladder symptoms, fatigue, spasticity, depression). A referral to an appropriate specialist should be made if required.

Women of childbearing age with MS should be advised that

- They should not defer disease-modifying drug treatment because they wish to have children in the future.

- Pregnancy does not increase the risk of worsening longterm disability.

- They should not suddenly stop their medications when they become pregnant, but should contact their MS team/general practitioner/midwife as soon as possible for advice.

- They should take vitamin D supplements: see Dobson et $a l^{5}$ for a discussion surrounding vitamin D; developed separately to the consensus group guidelines.

- Natural history studies in mainly untreated populations have suggested that although relapse frequency reduces during pregnancy, about a quarter of women will experience a relapse during the first 3 months postpartum. During a pregnancy year (ie, 9 months of pregnancy and 3 months postpartum), the risk of relapse is the same overall as that of a woman with MS who is not pregnant. ${ }^{6}$

- Relapses can be treated with corticosteroids during pregnancy.

- There may be an increased risk of having a relapse after use of gonadotropin-releasing hormone agonists for assisted conception; ${ }^{7}$ however, this should not deter women with MS from seeking assisted conception where this is indicated. In vitro fertilisation units should liaise with the MS team before prescribing assisted reproduction therapy. 


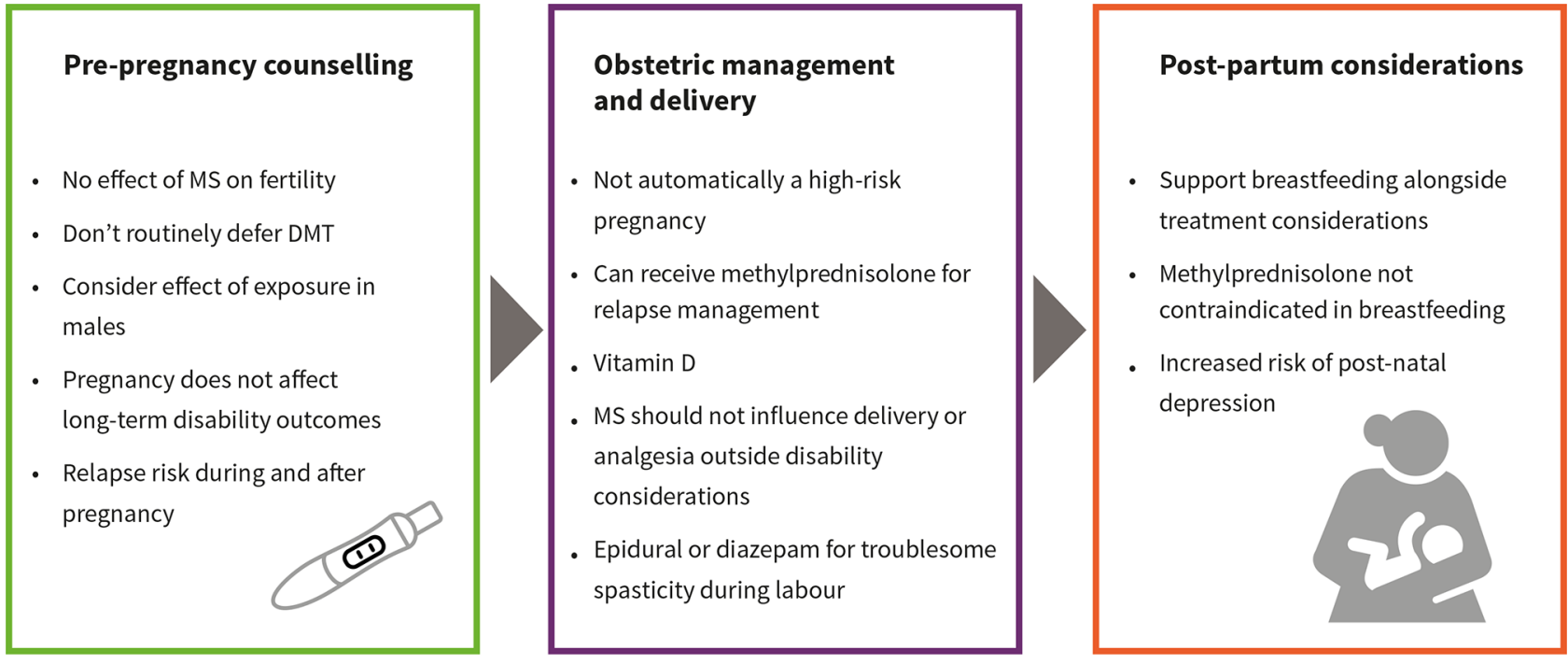

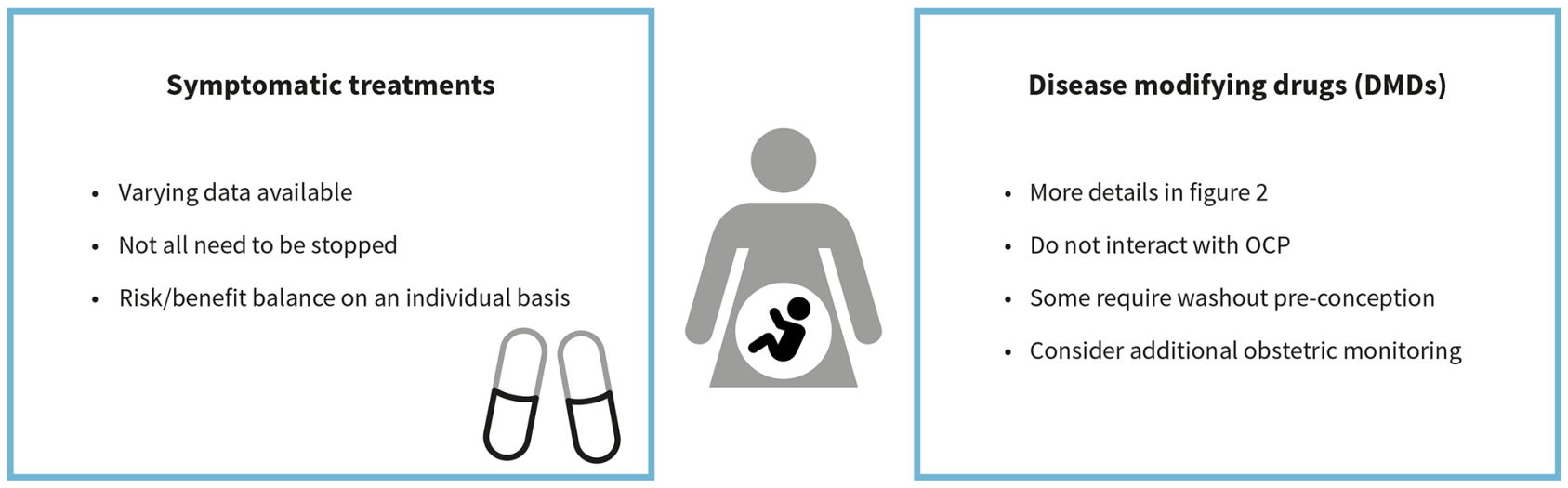

Figure 1 Infographic summarising topics to consider during prepregnancy counselling. DMT, disease modifying therapy; OCP, oral contraceptive pill.

- While many women find their MS symptoms improve during pregnancy, some symptoms may worsen, such as fatigue, balance and bladder symptoms, particularly in the latter stages of pregnancy. Bell's palsy and entrapment neuropathies, for example, carpal tunnel syndrome, meralgia paraesthetica, are more common in late pregnancy and may cause new symptoms that should be distinguished from a MS relapse.

- Having MS should not influence obstetric management, for example, whether they can have an epidural or spontaneous vaginal delivery, although for women with significant disability, for example, spasticity, this should be taken into consideration when planning obstetric care.

\section{Management during pregnancy}

- Women with MS should be advised to follow standard advice for all pregnant women, with particular emphasis on not smoking, performing pelvic floor exercises and taking recommended supplements.

- The MS team should be notified as soon as possible if a woman with MS becomes pregnant so they can provide appropriate support and liaise with her obstetric team.
- The woman with MS and her obstetric team should be made aware that having MS does not automatically mean the pregnancy should be judged as being high risk and antenatal care can usually be led by a midwife.

- No additional obstetric scans are required just because a woman has MS.

- Women with MS should be advised that urinary tract infections are more frequent in pregnancy and they should be provided with information to help them recognise the symptoms and advised to seek prompt treatment from their MS healthcare professional, obstetric team or general practitioner if concerned. The obstetric team should be made aware that people with MS who experience a urinary tract infection may experience a temporary worsening of their MS-related symptoms.

- Although relapses are less common during pregnancy, any women with MS suffering a disabling relapse should be offered intravenous methylprednisolone at the recommended dose used in MS, regardless of trimester, once an underlying infection, such as a urinary tract infection has been excluded. For very severe relapses that do not respond to corticosteroids, plasma exchange can be considered. 
- MRI is not contraindicated at any time during pregnancy $;^{8}$ however, gadolinium contrast media should be avoided where possible. ${ }^{8}$

- The MS team should consider a referral to an appropriate multidisciplinary team (potentially neurorehabilitation or MS-specific team depending on individual need) at any point during or after the pregnancy if the woman is experiencing problems that are likely to impact on her ability to care for the baby or herself. More specifically, if a woman has problems with spasticity or severe weakness of the pelvis and/or legs, she should be referred to a neuro-physiotherapist early in pregnancy to work collaboratively with her and her obstetric team to optimise labour and delivery.

Delivery and anaesthetic options

We recommend that:

- Troublesome increased spasticity during labour may be controlled using benzodiazepines and/or an epidural anaesthetic.

- MS should not influence the mode of delivery or analgesia/anaesthesia unless there is significant disability.

- Women with spinal cord involvement or loss of sensation below T11 may not be aware of the onset of labour. They should be taught to look for other symptoms indicative of the onset of labour including an increase in spasticity, gastrointestinal upset, flushing and back pain.

- Obstetric teams should be aware that for women with MS who have severe spinal cord disease, autonomic dysreflexia should be considered in the differential diagnosis for pre-eclampsia.

\section{Postpartum advice}

\section{We recommend that:}

- Women with MS should be encouraged to breastfeed, although it remains uncertain whether exclusive breastfeeding can reduce postpartum relapse rate. ${ }^{9}$

- Women who are breastfeeding should be advised to consider storing supplies of breast milk in the freezer for use in case of relapse-associated disability or severe fatigue or in case they need to be prescribed a medication not compatible with breastfeeding.

- There is no indication to stop breastfeeding if methylprednisolone is required to treat a postpartum relapse (other than if relapse-associated disability prevents safe breastfeeding). ${ }^{10}$

- There is no evidence to support regular methylprednisolone in the postpartum period to prevent relapses.

- Immunoglobulin should not be given to prevent postpartum relapses.

- Women with MS should continue to take vitamin D and their infants should be given vitamin $\mathrm{D}$ in line with standard advice.

- The neuro-multidisciplinary team should provide advice to the midwife and/or health visitor as required. The woman with MS may require additional support from her family/friends and the multidisciplinary team in the postpartum period. The MS team and midwife should help her to plan this in advance of the birth.

- There is evidence of an increased risk of postpartum depression in mothers and fathers with MS. ${ }^{11}$ The MS service, midwives, health visitors and GP should be aware of this and offer appropriate support as required. The MS team should ensure that the person with MS and their partner are aware of this, of the signs and symptoms of postpartum depression and how to get help if they are concerned at any time.

- There is insufficient evidence at present to recommend routine umbilical cord blood collection and banking for women with MS for future MS-related treatments, for example, stem cell therapies.

\section{DISEASE-MODIFYING DRUG SPECIFIC STATEMENTS (FIGURE 2)}

IFN-B and GA (Avonex, Betaferon, Extavia, Plegridy, Rebif, Copaxone)

A branded biosimilar GA (Brabio) is available but there are no pregnancy data available as yet.

- IFN-B/GA do not interact with hormonal contraception.

- There is no evidence from human studies of reduced fertility in men or women or of an increased congenital malformation or miscarriage rate associated with IFN-B/ GA.

- Previous concerns around an increased rate of spontaneous abortion associated with IFN-B ${ }^{12}$ have not been seen in larger registry studies. ${ }^{13}$

- The panel regard it as safe to continue these treatments at least up until conception, although at present only Copaxone holds a licence for use in pregnancy.

- There is no evidence of any need to consider termination if unplanned pregnancy occurs while taking IFN-B/GA.

- As relapse rate falls during pregnancy, continuing treatment with IFN-B/GA throughout pregnancy may be unnecessary for some women. However, for women who require treatment throughout pregnancy, there is no evidence that this will result in harm to the fetus. The benefits of this approach include a possible reduction in the frequency and severity of postpartum relapses, although it is difficult to predict in whom these will occur. The pros and cons of continuing treatment throughout pregnancy should be discussed with the woman with MS so that she can make an informed choice.

1213

- If IFN-B/GA are stopped during pregnancy, they will take several months to reach full efficacy when restarted and so may not reduce relapse rate during the first few months postpartum.

- While the summary of product characteristics say GA should be prescribed during breastfeeding only if its benefit is thought to outweigh potential risk and that IFNB should not be used while breastfeeding because of the potential for serious risk to the infant, the panel believe that the benefits of breastfeeding while on IFN-B/ GA outweigh any risk and should be encouraged. 
First line injectables

Copaxone/IFN-B
preparations
- Safe to continue until conception
- No evidence of harm to fetus
- If stopped, $3 / 12$ to reach full
efficacy post-partum
- Benefits of breastfeeding on
treatment outweigh risks
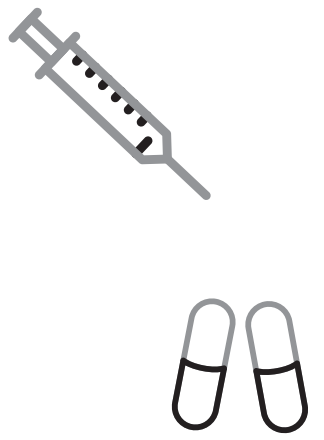

\section{First line oral}

\begin{tabular}{l}
\hline Teriflunomide \\
- Teratogenic animal studies \\
- Potential exposure in females \\
via transfer in seminal fluid \\
2 years washout or accelerated \\
elimination \\
- Unplanned pregnancies: \\
accelerated elimination and \\
high risk \\
- Breastfeeding contraindicated \\
\hline Dimethyl Fumarate \\
- Consider effect of Gl upset on OCP \\
- Limited data in pregnancy \\
- Breastfeeding contraindicated
\end{tabular}

\section{Alemtuzumab}

- Can try to conceive 4/12 after

course of alemtuzumab

- Monitor for autoimmune disease during pregnancy

- Irradiated blood products

- Do not give when breastfeeding

\section{Cladribine}

- Teratogenic in $\mathrm{M}$ and $\mathrm{F}$

- Avoid pregnancy for $6 / 12$ after treatment course

- Breastfeeding contraindicated

- Breastfeeding contraindicated

Ocrelizumab
- Avoid pregnancy for $12 / 12$
- Unplanned pregnancy: immediately
stop; high risk
- Breastfeeding contraindicated

Figure 2 Infographic summarising advice about the use of disease-modifying drugs during pregnancy and in breastfeeding. IFN-B, interferon beta.

\section{Natalizumab (Tysabri)}

- Women with MS should be advised that natalizumab does not interact with hormonal contraception.

- Men and women with MS should be advised that there is no evidence in human studies of natalizumab resulting in reduced fertility or congenital malformations.

- The MS team should discuss the pros and cons of stopping vs continuing treatment with natalizumab during pregnancy with the woman with MS (as recommended in the summary of product characteristics), ideally before conception or alternatively as soon as the woman informs the MS team she is pregnant. ${ }^{14}$ This discussion should include:

- Women eligible for natalizumab have rapidly evolving severe MS and the beneficial hormonal effects of pregnancy on reducing relapse rate may not be sufficient alone to adequately control their MS disease activity. $^{3}$

- There is a risk of rebound, that is, a severe increase in relapse rate, associated with stopping natalizumab. Rebound normally occurs 12-16 weeks after stopping treatment. ${ }^{3}$

- While there is still only limited experience, there have been to date no specific pattern of birth defects observed with receiving natalizumab during pregnancy to suggest it is teratogenic. ${ }^{15}$ Recent evidence suggests a possible increased risk of miscarriage associated with natalizumab treatment but this was not statistically significantly different to the control population. ${ }^{16}$

- Natalizumab does not cross the placenta during the first trimester, but it is actively transported during the second and third trimesters. To minimise fetal exposure, the panel recommends giving the last dose during pregnancy at approximately 34 weeks and restarting soon after birth; within 8-12 weeks after the last dose wherever possible to avoid rebound disease activity. Exposure late in pregnancy has been associated with minor, selflimiting haematological abnormalities in infants. ${ }^{17}$ The frequency of natalizumab infusions can be reduced to 8-weekly throughout pregnancy to reduce exposure, in line with a recent study that showed this is unlikely to diminish its effectiveness. ${ }^{18}$ However, this extended dosing regimen is unlicensed.

- We recommend that routine MR brain imaging monitoring for progressive multifocal leukoencephalopathy should continue during pregnancy as required. There 
have been no congenital cases of progressive multifocal leukoencephalopathy described to date.

- Natalizumab is transferred to/crosses into breast milk and the amount increases with time. ${ }^{19}$ However, there are significant benefits of breastfeeding. Whilst the SPC for Natalizumab states that it should be discontinued whilst breastfeeding, the panel believed that as the oral bioavailability is negligible, any transfer into breast milk is unlikely to result in systemic absorption by the baby.

\section{Fingolimod (Gilenya)}

- Women with MS should be advised that fingolimod does not interact with hormonal contraception.

- Men and women with MS should be advised that there is no evidence in human studies of fingolimod resulting in reduced fertility or increased congenital malformation or miscarriage rates.

- There is limited information about the safety of fingolimod in pregnancy and women with MS should be advised to use effective contraception while taking it.

- Women with MS planning to become pregnant should be advised to stop fingolimod at least 2 months before conception and should discuss alternative treatments.

- Patients should be advised to stop the treatment immediately in the event of an unplanned pregnancy.

- Pregnancies in women on fingolimod should be treated as high-risk and we recommend referral to an obstetrician for consideration of a fetal medicine scan.

- Women should not take fingolimod while breastfeeding.

\section{Teriflunomide (Aubagio)}

- Women with MS should be advised that teriflunomide does not interact with hormonal contraception.

- Men should be advised that teriflunomide can reduce sperm counts and male-to-female transfer of sperm may result in low levels of teriflunomide being present in the female.

- Women wishing to conceive who have received teriflunomide should stop it and undergo the accelerated elimination procedure, as teriflunomide has a very long enterohepatic circulation. They should continue to use contraception for at least 6 weeks after serum drug concentrations have been shown to be less than 0.02 $\mathrm{mg} / \mathrm{L}$ on two occasions at least 14 days apart.

- Teriflunomide is teratogenic in animals at equivalent doses to those given to humans and the panel advise caution in prescribing this to women of childbearing age.

- Women with MS should be advised that they must use effective contraception while on this treatment and for 2 years after stopping unless they have undergone the accelerated elimination procedure.

- Women with MS who are taking teriflunomide and wish to stay on a disease-modifying drug should be advised by their MS team to switch to an alternative treatment if they wish to become pregnant. They will then need to complete the accelerated elimination procedure or continue to use contraception for at least 6 weeks after serum drug concentrations have been shown to be less than $0.02 \mathrm{mg} / \mathrm{L}$ on two occasions at least 14 days apart.

- We recommend that women who have an unplanned pregnancy while taking teriflunomide should undergo the accelerated elimination procedure to reduce circulating levels of teriflunomide (as set out in summary of product characteristic documentation) as soon as possible.

- Pregnancies in women on teriflunomide should be treated as high risk and we recommend referral to an obstetrician for consideration of a fetal medicine scan.

- Women should not take teriflunomide while they are breastfeeding.

\section{Dimethyl fumarate (Tecfidera)}

- Women with MS should be advised that dimethyl fumarate does not reduce the effectiveness of hormonal contraception. However, gastrointestinal upset can occur within the first few weeks of treatment and additional contraceptive measures are advisable in case of interference with absorption of the contraceptive pill/ oral contraceptives (both combined oestrogen and progesterone, and progesterone only pill).

- Men and women with MS should be advised that there is no evidence in human studies of dimethyl fumarate resulting in reduced fertility or increased congenital malformation or miscarriage rates.

- There are limited data about the safety of dimethyl fumarate in pregnancy and we recommend that women with MS should use effective contraception while receiving this therapy and if they do become pregnant they should only continue treatment if the potential benefit justifies the potential risk to the fetus.

- The MS team should discuss switching to an alternative treatment if a woman with MS taking dimethyl fumarate wishes to become pregnant.

- It is not known if dimethyl fumarate or its metabolites are excreted in breast milk and, if they are, what harm this might cause to the baby. The panel recommend that women with MS do not take dimethyl fumarate while breastfeeding.

\section{Ocrelizumab (Ocrevus)}

- Women with MS should be advised that ocrelizumab is not known to interact with the hormonal contraception.

- The manufacturers recommend that women should use contraception while receiving ocrelizumab and for 12 months after the last infusion.

- Ocrelizumab is a humanised monoclonal IgG antibody; immunoglobulins are known to cross the placenta, thus fetal exposure is likely if the drug is given during pregnancy. There are limited data regarding the use of ocrelizumab in pregnancy. Specifically, no B-cell count data have been collected in infants exposed to ocrelizumab, and the potential duration of B-cell depletion in infants is unknown. Transient peripheral B-cell depletion and lymphopenia have been reported in infants born to mothers exposed to other anti-CD20 monoclonal 
antibodies during pregnancy (in particular rituximab ${ }^{20}$ ); it would seem reasonable to assume that the same may be true for ocrelizumab.

- Due to the potential for B-cell depletion in infants of mothers who have been exposed to ocrelizumab during pregnancy, infants should be monitored for B-cell depletion. Vaccinations with live and live-attenuated vaccines should be postponed until the infant's B-cell count has recovered.

- It is unknown whether ocrelizumab or its metabolites are excreted in breast milk. Animal data have shown excretion of ocrelizumab in milk. Women should be advised not to receive ocrelizumab while breastfeeding.

\section{Alemtuzumab (Lemtrada)}

- Serum concentrations of alemtuzumab are low or undetectable approximately 30 days after a course of treatment. The manufacturers recommend women should use effective contraception for 4 months following a course of treatment with alemtuzumab. If pregnancy does occur within a month of treatment, increased obstetric monitoring is advised.

- Women with MS should be advised that alemtuzumab does not impair the effectiveness of hormonal contraception.

- For 4 years after a cycle of alemtuzumab, women are at risk of autoimmune thyroid disease (37\%) and immune thrombocytopenic purpura (1\%), Goodpasture's syndrome $(0.1 \%)$ and other autoimmune diseases. ${ }^{21}$ These may occur during pregnancy and affect both the mother and fetus (for instance, neonatal thyrotoxicosis). Early identification is important with continuation of the routine safety monitoring of monthly full blood count and renal function, with urine dipstick, and 3 monthly thyroid function tests. The women's obstetric team should be informed of these risks and appropriate specialist advice sought if they are identified.

- If the woman with MS has thyroid disease during pregnancy (whether controlled or not), the fetus should receive specialist monitoring; there are obstetric protocols for this and the obstetricians should be informed.

- A fatal case of graft-versus-host disease following blood transfusion has been reported in an oncology patient treated with alemtuzumab, although this patient had also received fludarabine. ${ }^{22}$ Where possible, irradiated blood products should be used in patients who have received alemtuzumab.

- Women with MS should not be treated with alemtuzumab during pregnancy or while breastfeeding.

\section{Cladribine (Mavenclad)}

- Cladribine is incorporated into DNA strands and inhibits DNA synthesis and repair. It is embryolethal in female mice and teratogenic when administered to either female or male mice.

- Women taking cladribine should be advised that it does not change the effectiveness of hormonal contraception. However, given cladribine's mechanism of action of its potential for serious risk to the fetus, the manufacturers recommend that women using hormonal contraception should add a barrier method of contraception during cladribine treatment and for at least 4 weeks after the last dose in each treatment year. Women should be advised not to become pregnant for at least 6 months following a course of cladribine.

- As cladribine interferes with DNA synthesis, adverse effects on gametogenesis could be expected. Therefore, male partners must take precautions to prevent pregnancy of their partner during cladribine treatment and for at least 6 months after the last dose.

- Increased numbers of non-motile sperm were seen in animals given cladribine; however, this was not associated with a decrease in fertility of these animals; there are no human data on fertility.

- Women who become pregnant following cladribine treatment should stop any further treatment until after pregnancy. Studies in animals have shown reproductive toxicity associated with cladribine.

- In keeping with the advice in the summary of product characteristics, where possible irradiated blood products should be used in patients who have received cladribine.

- It is not known whether cladribine is excreted in human milk. Given the potential for serious adverse effects in breast-fed infants, breastfeeding is contraindicated during treatment with cladribine and for 1 week after the last dose.

Symptomatic treatments

There is a wide range of symptomatic treatments used in MS, and we plan an in-depth discussion on their use in a subsequent review.

There have been National Institute for Health and Care Excellence (NICE) clinical knowledge summaries produced for the use of antidepressant and antiepileptic medications prenatally and postnatally, providing easily accessible information on how to counsel women both before pregnancy and identifying those in whom it may be indicated during pregnancy (https://cks.nice.org.uk/depression-antenatal-andpostnatal; https://cks.nice.org.uk/epilepsy\#!scenario: 3). Additionally, symptomatic treatments used in rheumatological diseases (including simple analgesics and chronic pain treatments) have been recently comprehensively reviewed in the rheumatology literature. ${ }^{23}$

\section{CONCLUSION}

There are increasing data that delaying starting disease-modifying drugs and/or routinely stopping all disease-modifying drugs prepregnancy or immediately postconception is associated with increased relapse rate during pregnancy. This can have significant negative effects on long-term disability in women with MS. Consideration should be given regarding the potential for future pregnancies when starting women of childbearing age on disease-modifying drugs. Counselling around disease-modifying drugs and symptomatic 
medication in pregnancy needs to happen before the woman stops contraception where possible. There are potential issues around pregnancy in women with advanced MS or significant cognitive impairment, which should be discussed on an individual patient basis and are beyond the scope of these guidelines.

First-line injectable treatments, such as GA and beta interferon, may be continued during pregnancy. Patients taking natalizumab will usually have had highly active disease before treatment, and serious consideration should be given to continuing treatment during pregnancy given the significant risk of disease reactivation and/or rebound on stopping treatment. Pregnancy is not recommended for 4 months following alemtuzumab and for 6 months following treatment with cladribine; however after this time there are no contraindications. It is not recommended to try to conceive for 12 months after receiving ocrelizumab; because this medication is given on a 6 -monthly basis, family planning must be considered when initiating and prescribing. Teriflunomide is contraindicated in pregnancy, and dimethyl fumarate and fingolimod should be avoided where possible.

An independent pregnancy register of all women with MS who become pregnant, with prospective data collection on all medications and pregnancy-related outcomes is much needed-the epilepsy pregnancy register has greatly informed the care of women on antiepileptic medication. Currently, in the UK, there are MS pregnancy registers specifically focussing on patients taking dimethyl fumarate, teriflunomide and alemtuzumab. While neurologists should encourage their patients taking these medications to contribute to these registers, longer term developmental effects, such as those seen in the children of women taking valproate, are likely only to be detected via large registry studies. Information regarding the safety of medications in pregnancy is constantly evolving and being updated as new data are published; as such neurologists should be aware of where to find information and to support the

\section{Key Points}

- Treatment with disease-modifying drugs should not be delayed until a woman with multiple sclerosis (MS) has completed her family.

- Consider the possibility of pregnancy when prescribing to all women with MS of childbearing age.

- Relapse rate reduces naturally during pregnancy, and so many women with MS choose to stop diseasemodifying drugs when they become pregnanthowever, for those with very active MS, treatment throughout pregnancy should be considered.

- If relapse occurs, corticosteroids can be given during pregnancy and while breastfeeding.

- Having MS does not automatically make pregnancy high risk and should not in itself limit birthing options. creation of joint neuro-obstetric clinics to allow for the best multidisciplinary care for our pregnant patients.

Acknowledgements These guidelines were developed by a working group that was composed of: Neurologists: Pooja Dassan, Peter Brex, Martin Lee, Helen Ford, Orla Gray, Gavin Giovannoni, Alasdair Coles; MS nurses: Pauline Shaw, Catherine Womersley, Megan Roberts; Obstetrician: Mandish K Dhanjal; Midwifery: Carmel Bagness; Pharmacy: Aoife Sheilds; Obstetric Physician: Catherine Nelson-Piercy. Dr Surabhi Nanda provided additional input into manuscript.

Contributors PD, PAB and GG conceived the idea of the guidelines. PAB, PD and MR developed the working group discussion into guidelines for the review process and revised these in response to initial review. RD, PAB, PD and CN-P developed the working group recommendations into the guideline format. RD drafted the initial paper for dissemination and incorporated further feedback from all authors and the wider working group. All authors provided input into the final manuscript.

Funding The project was supported by the MS Trust.

Competing interests None declared.

Patient consent Not required.

Provenance and peer review Provenance and peer review. Commissioned. Externally peer reviewed by Emma Tallantyre, Cardiff, UK.

Addendum Following the publication of 'UK consensus on pregnancy in multiple sclerosis: ABN guidelines', new information has become available. Further information is available athttps://pn.bmj.com/content/19/2/106.responses\#ukconsensus-on-pregnancy-in-multiple-sclerosis-an-update.

\section{REFERENCES}

1 Anon. ePosters. Mult Scler 2017;23(3_suppl):680-975.

2 Comi G, Radaelli M, Soelberg Sørensen P. Evolving concepts in the treatment of relapsing multiple sclerosis. Lancet 2017;389:1347-56.

3 Portaccio E, Moiola L, Martinelli V, et al. Pregnancy decision-making in women with multiple sclerosis treated with natalizumab: II: Maternal risks. Neurology 2018;90:e832-e839.

4 Franklin GM, Tremlett H. Multiple sclerosis and pregnancy: what should we be telling our patients? Neurology 2009;73:1820-2.

5 Dobson R, Cock HR, Brex P, et al. Vitamin D supplementation. Pract Neurol 2018;18:35-42.

6 Confavreux C, Hutchinson M, Hours MM, et al. Rate of pregnancy-related relapse in multiple sclerosis. Pregnancy in Multiple Sclerosis Group. N Engl J Med 1998;339:285-91.

7 Michel L, Foucher Y, Vukusic S, et al. Increased risk of multiple sclerosis relapse after in vitro fertilisation. J Neurol Neurosurg Psychiatry 2012;83:796-802.

8 Ray JG, Vermeulen MJ, Bharatha A, et al. Association Between MRI Exposure During Pregnancy and Fetal and Childhood Outcomes. JAMA 2016;316:952-61.

9 Hellwig K, Rockhoff M, Herbstritt S, et al. Exclusive Breastfeeding and the Effect on Postpartum Multiple Sclerosis Relapses. JAMA Neurol 2015;72:1132-8.

10 Boz C, Terzi M, Zengin Karahan S, et al. Safety of IV pulse methylprednisolone therapy during breastfeeding in patients with multiple sclerosis. Mult Scler 2018;24:1205-11.

11 Razaz N, Tremlett H, Marrie RA, et al. Peripartum depression in parents with multiple sclerosis and psychiatric disorders in children. Mult Scler 2016;22:1830-40. 
12 Sandberg-Wollheim M, Frank D, Goodwin TM, et al. Pregnancy outcomes during treatment with interferon beta-1a in patients with multiple sclerosis. Neurology 2005;65:802-6.

13 Hellwig K, Geissbuehler Y, Sabidó M, et al. Cumulative data from the European interferon beta pregnancy registry. Neurology 2018;90.

14 Marrie RA. Maternal and fetal risks of natalizumab exposure in utero: A fine balance. Neurology 2018;90:443-4.

15 Friend S, Richman S, Bloomgren G, et al. Evaluation of pregnancy outcomes from the Tysabri ${ }^{\circledR}$ (natalizumab) pregnancy exposure registry: a global, observational, follow-up study. BMC Neurol 2016;16:150.

16 Portaccio E, Annovazzi P, Ghezzi A, et al. Pregnancy decisionmaking in women with multiple sclerosis treated with natalizumab: I: Fetal risks. Neurology 2018;90:e823-e831.

17 Haghikia A, Langer-Gould A, Rellensmann G, et al. Natalizumab use during the third trimester of pregnancy. JAMA Neurol 2014;71:891-5.

18 Zhovtis Ryerson L, Frohman TC, Foley J, et al. Extended interval dosing of natalizumab in multiple sclerosis. J Neurol Neurosurg Psychiatry 2016;87:885-9.
19 Baker TE, Cooper SD, Kessler L, et al. Transfer of natalizumab into breast milk in a mother with multiple sclerosis. J Hum Lact 2015;31:233-6.

20 Chakravarty EF, Murray ER, Kelman A, et al. Pregnancy outcomes after maternal exposure to rituximab. Blood 2011;117:1499-506.

21 Coles AJ, Cohen JA, Fox EJ, et al. Alemtuzumab CARE-MS II 5-year follow-up: Efficacy and safety findings. Neurology 2017;89:1117-26.

22 Lin TS, Donohue KA, Byrd JC, et al. Consolidation therapy with subcutaneous alemtuzumab after fludarabine and rituximab induction therapy for previously untreated chronic lymphocytic leukemia: final analysis of CALGB 10101. J Clin Oncol 2010;28:4500-6.

23 Flint J, Panchal S, Hurrell A, et al. BSR and BHPR guideline on prescribing drugs in pregnancy and breastfeeding-Part II: analgesics and other drugs used in rheumatology practice. Rheumatology 2016;55:1698-702. 